\title{
Octonacci photonic quasicrystals
}

\author{
E.R. Brandão ${ }^{a}$, C.H. Costa ${ }^{\text {b,a }}$, M.S. Vasconcelos ${ }^{\text {c,* }}$, D.H.A.L. Anselmo ${ }^{a}$, V.D. Mello ${ }^{d}$ \\ ${ }^{a}$ Departamento de Física Teórica e Experimental, Universidade Federal do Rio Grande do Norte, 59600-900 Natal, RN, Brazil \\ ${ }^{\mathrm{b}}$ Universidade Federal do Ceará, Campus Avançado de Russas, 62900-000 Russas, CE, Brazil \\ ${ }^{\mathrm{c}}$ Escola de Ciências e Tecnologia, Universidade Federal do Rio Grande do Norte, 59072-970 Natal, RN, Brazil \\ ${ }^{\mathrm{d}}$ Departamento de Física, Universidade do Estado do Rio Grande do Norte, Mossoró, RN 59625-620, Brazil
}

\section{A R T I C L E I N F O}

\section{Article history:}

Received 6 March 2015

Received in revised form 10 April 2015

Accepted 23 April 2015

Available online 9 May 2015

\section{Keywords:}

Photonic crystals

Photonic band gaps

Quasiperiodic system

Optical properties

\begin{abstract}
A B S T R A C T
We study theoretically the transmission spectra in one-dimensional photonic quasicrystals, made up of $\mathrm{SiO}_{2}(A)$ and $\mathrm{TiO}_{2}(B)$ materials, organized following the Octonacci sequence, where the $n$ th-stage of the multilayer $S_{n}$ is given by the rule $S_{n}=S_{n-1} S_{n-2} S_{n-1}$, for $n \geqslant 3$ and with $S_{1}=A$ and $S_{2}=B$. The expression for transmittance was obtained by employing a theoretical calculation based on the transfer-matrix method. For normally incident waves, we observe that, for a same generation, the transmission spectra for transverse electric (TE) and transverse magnetic (TM) waves are equal, at least qualitatively, and they present a scaling property where a self-similar behavior is obtained, as an evidence that these spectra are fractals. The spectra show regions where the omnidirectional band gaps emerges for specific generations of Octonacci photonic structure, except to TM waves. For TE waves, we note that all of them have almost the same width, for different generations. We also report the localization of modes as a consequence of the quasiperiodicity of the heterostructure.
\end{abstract}

(c) 2015 Elsevier B.V. All rights reserved.

\section{Introduction}

Since the pioneer works of Yablonovitch [1] and John [2] in which the photonic crystals (PCs) were proposed, many experimental and theoretical investigations have been devoted to the understanding of the physical properties of these systems (for a review, we recommend the Refs. [3-5]). PCs are structures characterized by the periodic variation of their refractive index, allowing the appearance of the so-called photonic band gaps (PBGs) [6,7], and, because of this, they have the property to control the light propagation, leading to a new age for optical devices [8,9]. Some very interesting applications of PBGs are in the fields of waveguides based on PC [10], optical reflectors [11], etc.

On the other hand, the discovery of quasicrystals, in 1982, by Shechtman et al. [12] has fired up a new field in condensed matter physics, resulting in many theoretical and experimental works (for an up to date review of this field, see Refs. $[13,9])$. For such amazing discovery, in 2011, Shechtman was awarded with the Nobel Prize in Chemistry [14]. Historically, the concept of quasiperiodicity was first introduced by the mathematician H.A. Bohr, in 1926, during his studies about almost periodic functions [15]. Decades later, in 1970, the works of Penrose and Ammann demonstrated

\footnotetext{
* Corresponding author.

E-mail address: mvasconcelos@ect.ufrn.br (M.S. Vasconcelos).
}

that it is possible to fill a non-Euclidean gapless space in a nonperiodic way. These analysis can be considered as the foundations of the field of quasiperiodic structures (or tillings) [16]. Penrose has proposed a tiling of the plane, which today is well known as the Penrose tiling, with two rhombic tiles which are arranged according to specific rules [17]. In the same decade, Ammann found tilings with an eightfold symmetry filling the gapless plane $[18,19]$. Today, many different tilings are known which cannot only fill the two-dimensional space quasiperiodically, but also three and higher dimensions, like the three-dimensional Ammann-KramerNeri tiling [20] or the zonotiles [21].

For a quasiperiodic arrangement, which displays an important role for promising technological applications of aperiodic systems [22], the photonic crystals can be called photonic quasicrystals (PQCs) [23,24], which are defined like the PCs: the difference is in the spatial distribution of the refraction indexes, where they are organized in a quasiperiodic fashion [25], obeying a mathematical rule, which, here, is the Octonacci substitutional sequence. The studies of photonic quasicrystals started with the pioneer works of Kohomoto et al. [26], where they proposed a one-dimensional multilayered system arranged in accordance to a Fibonacci quasiperiodic rule, with a quarter wavelength condition. Since that, many theoretical and experimental works have been published in this field (see Refs. $[27,28]$ and references therein). It has been recognized that in addition to crystalline and amorphous materials, 
there exists a third intermediate class known as "deterministic aperiodic" structures, which can be generated by a substitution rule based on two or more [29,30] building blocks that exhibit long-range order, which does not have translational symmetry. Also, it has been shown that these structures exhibit properties of self-similarity in their spectra [13]. In a general way, this new class of structures can be classified into two groups: quasicrystals and all other deterministic aperiodic structures. Quasicrystals therefore represent a special class of deterministic aperiodic structures. A more precise and updated definition of quasicrystals with dimensionality $n(n=1,2$ or 3$)$ is that in addition to their possible generation by a substitution process, they can also be formed from a projection of an appropriate periodic structure in a higher dimensional space $m D$, where $m>n$ [24]. In contrast, structures that are part of the other deterministic structures cannot be constructed in such a manner. For example, in one dimension (1D), quasicrystalline structures include the Fibonacci sequence and their generalizations [31-34]. Examples of aperiodic structures that differ from quasicrystals are systems that obey the Thue-Morse, double-period [35] and Rudin-Shapiro [36] sequences.

The aim of this work is to investigate the transmission spectra of a obliquely incident light beam from a transparent medium into a multilayer photonic structure composed of $\mathrm{SiO}_{2}$ and $\mathrm{TiO}_{2}$ layers arranged in accordance to the quasiperiodic Octonacci sequence, which describes the arrangement of spacing of the Ammann quasilattice (8-grid), namely, the octagonal Ammann-Becker tiling [16].

This paper is organized as follow: in Section 2, we present the Octonacci sequence, as well as the theoretical model to calculate the transmission spectra of TE and TM waves in PQCs, which is based on the well-known transfer-matrix method. In Section 3, we present some numerical results for the transmittance spectra as function of frequency and incident angle of the wave. We could observe the expected self-similar behavior of the transmission spectra for normally incident waves and the appearance of the omnidirectional photonic band gaps, that are well defined only for TE waves. The conclusions are presented in Section 4. Finally, we present our acknowledgements.

\section{Theoretical model: Octonacci sequence and transfer-matrix method}

An Octonacci multilayer photonic structure can be defined by the growth, by juxtaposition, of two building blocks $A\left(\mathrm{SiO}_{2}\right)$ and $B\left(\mathrm{TiO}_{2}\right)$, where the $n$ th-stage of the multilayer $S_{n}$ is given iteratively by the rule [37]

$S_{n}=S_{n-1} S_{n-2} S_{n-1}$,

for $n \geqslant 3$, with $S_{1}=A$ and $S_{2}=B$. The number of the building blocks increases according to Pell number $P_{n}=2 P_{n-1}+P_{n-2}$ (with $P_{1}=1, P_{2}=1$ and $n \geqslant 3$ ). The number of the building blocks of the materials $A$ and $B$ in $n$-th stage is given by $n_{A}+n_{B}=P_{n}$. Here, $n_{A}=P_{n}$ and $n_{B}=P_{n-1}$, with $P_{1}=0, P_{2}=1$ and $n \geqslant 3$. The number of building blocks $B$ divided by the number of building blocks $A$, in the limit $n \rightarrow \infty$, is $\tau=1+\sqrt{2}$. Another way to obtain this sequence is by using the follow recurrence rule: $A \rightarrow B, B \rightarrow B A B$. Note that this sequence is classified as Pisot-Vijayaraghavan (PV), when we take the negative eigenvalue of the substitution matrix [38], i.e., $\sigma^{-}=1-\sqrt{2}$, with $\left|\sigma^{-}\right| \leqslant 1$. On the other hand, differently of the Fibonacci case, the ratio $\tau$ between the number of building blocks $A$ and building blocks $B$ is different of the positive eigenvalue $\sigma^{+}=1+\sqrt{2}$, as usually is found in the Fibonacci generalizations $[25,39,40]$.

Although several theoretical techniques have been used to study the transmission spectra in these structures, like spectral analysis [41], in the present work we make use of the transfer-matrix approach to analyze them (for a review see Ref. [13]). On the present section we have two goals: first, we want to investigate the behavior of the normally incident light when it pass through a Octonacci photonic layered system, considering the central wavelength $\lambda_{0}=700 \mathrm{~nm}$, where the central gap will appear [35]. Second, we intend to investigate the influence of the oblique incidence in such systems, by looking for frequency regions where the band gaps are independent of the incident angle and/or of the wave polarization, i.e., the so-called omnidirectional photonic band gaps.

We consider that an electromagnetic radiation of frequency $\omega$ and $s$-polarization (TE waves), or $p$-polarization (TM waves), is incident from a transparent medium $C$ at an arbitrary angle $\theta_{C}$ with respect to the normal direction of the layered system (see Fig. 1). The layered system is formed by an array of slabs of the different materials $A$ and $B$. The reflectance $R$ and the transmittance $T$ coefficients are given, respectively, by [36],

$R=\left|\frac{M_{n}(2,1)}{M_{n}(1,1)}\right|^{2} \quad$ and $\quad T=\left|\frac{1}{M_{n}(1,1)}\right|^{2}$,

where $M_{n}(i, j)$, with $i, j=1,2$, are the elements of the optical transfer-matrix $M_{n}$, for the $n$-th generation of the sequence, and which links the coefficients of the electromagnetic fields in the region $z<0$ to those in the region $z>L$, where $L$ means the size of the quasiperiodic structure.

In order to illustrate the method to calculate the optical transfer-matrices, let us consider, firstly, the case in which we have two different dielectric media $A$ and $B$ with thicknesses $d_{A}$ and $d_{B}$, and non-dispersive refractive indexes $n_{A}$ and $n_{B}$, respectively, and a third transparent medium $C$, with refractive index $n_{C}$ that surrounds the quasiperiodic unit cell composed by the binary chain $A$ and $B$ (see Fig. 1). The transmission of an obliquely incident light wave across the interfaces $\alpha \rightarrow \beta(\alpha, \beta=A, B$ or $C)$ is represented by the transmission matrix (for more details, see Ref. [42])

$M_{\alpha \rightarrow \beta}^{s}=\frac{1}{2}\left[\begin{array}{ll}1+\frac{k_{z \beta}}{k_{z \alpha}} & 1-\frac{k_{z \beta}}{k_{z \alpha}} \\ 1-\frac{k_{z \beta}}{k_{z \alpha}} & 1+\frac{k_{z \beta}}{k_{z \alpha}}\end{array}\right]$,

with

$k_{z \eta}=\left[\left(\frac{n_{\eta} \omega}{c}\right)^{2}-k_{x}^{2}\right]^{1 / 2}, \quad(\eta=A, B$ or $C)$

and

$k_{x}=\frac{n_{C} \omega}{c} \sin \left(\theta_{C}\right)$,

for TE or s-polarization waves. Here we have used the fact the all $x$-components of the wavevector are equal due to Snell's laws, i.e., $k_{x}=k_{x A} \sin \theta_{A}=k_{x B} \sin \theta_{B}=k_{C} \sin \theta_{C}$, with $k_{C}=n_{C} \omega / c$. Beside of this, for TM or $p$-polarization waves crossing the interface $\alpha \mid \beta$, from medium $\alpha$ to $\beta$ one, we have

$M_{\alpha \rightarrow \beta}^{p}=\frac{1}{2} \frac{n_{\beta}}{n_{\alpha}}\left[\begin{array}{cc}1+\frac{k_{z \beta}}{k_{z \alpha}} \frac{n_{\alpha}}{n_{\beta}} & 1-\frac{k_{z \beta}}{k_{z \alpha}} \frac{n_{\alpha}}{n_{\beta}} \\ 1-\frac{k_{z \beta}}{k_{z \alpha}} \frac{n_{\alpha}}{n_{\beta}} & 1+\frac{k_{z \beta}}{k_{z \alpha}} \frac{n_{\alpha}}{n_{\beta}}\end{array}\right]$.

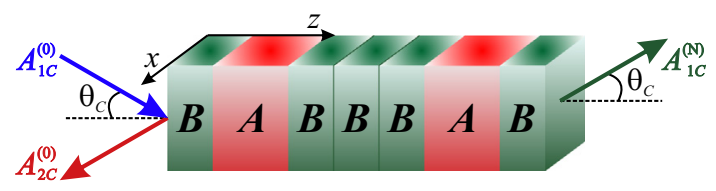

Fig. 1. Schematic representation for the geometry of the Octonacci quasiperiodic multilayer system considered in this work, more precisely, for sequence $S_{4}=[B|A| B|B| B|A| B]$, with $P_{4}=7 . L$ is the size of the whole superlattice structure. 
(a)

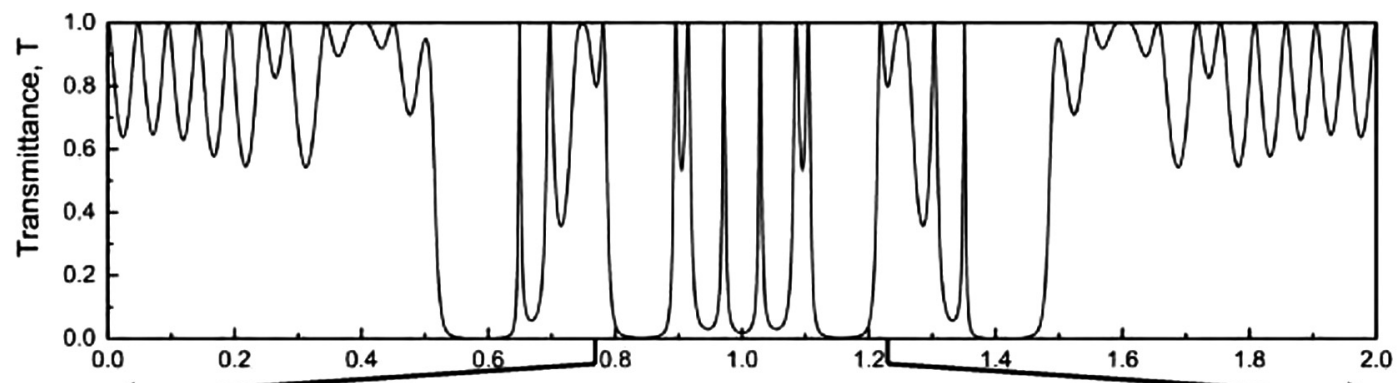

(b)

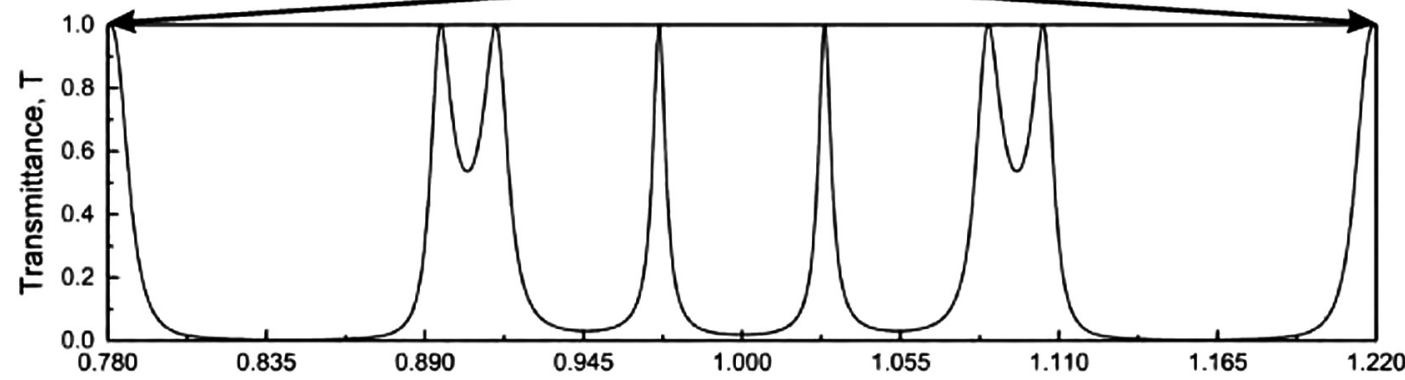

(c)

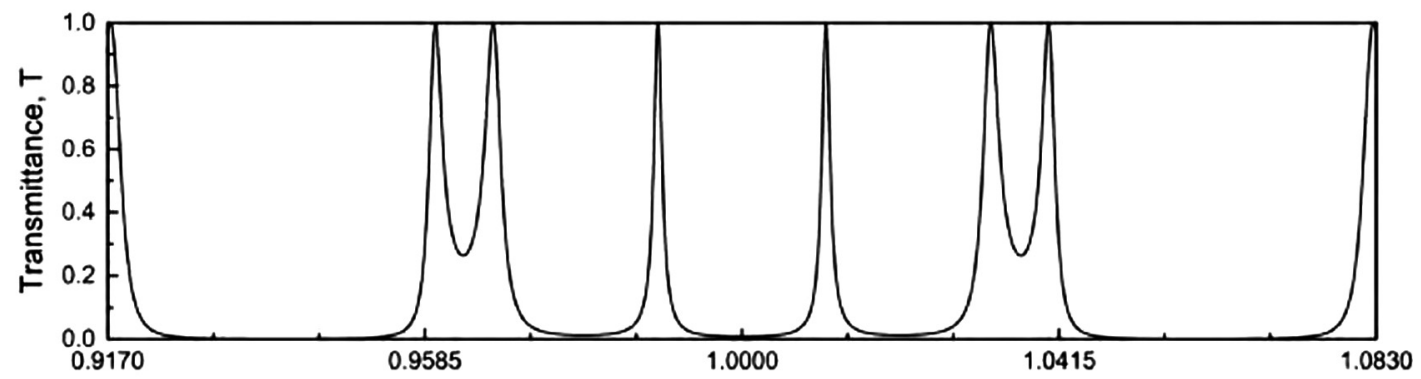

(d)
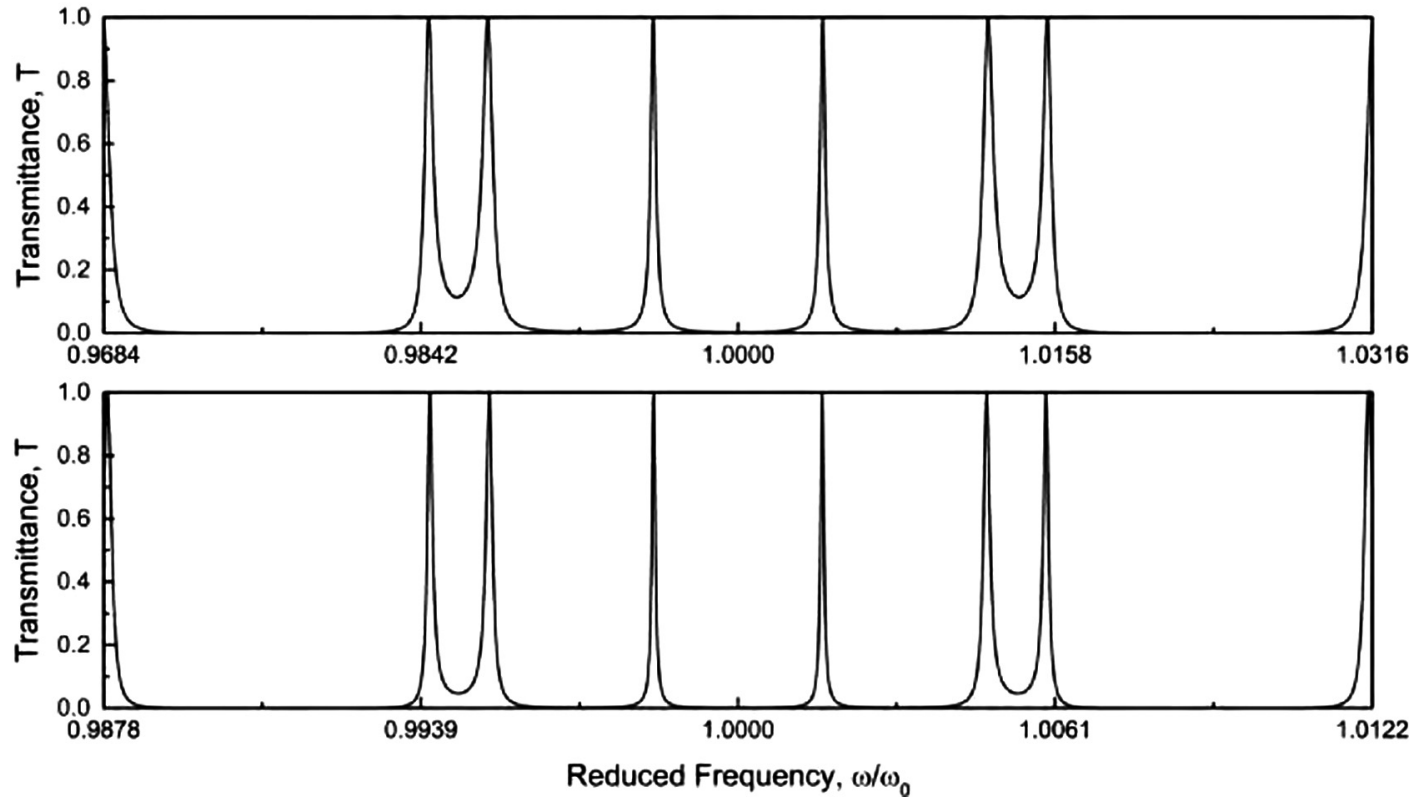

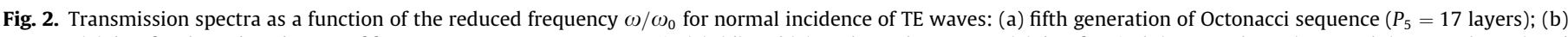

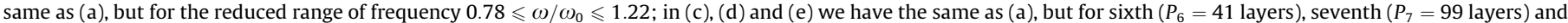
eighth $\left(P_{8}=239\right.$ layers) generations of Octonacci sequence, with the respective scales $0.917 \leqslant \omega / \omega_{0} \leqslant 1.083,0.9684 \leqslant \omega / \omega_{0} \leqslant 1.0316$, and $0.9878 \leqslant \omega / \omega_{0} \leqslant 1.0122$.

On the other hand, the propagation of the light wave within of a layer $\gamma(\gamma=A$ or $B)$, for both TE and TM waves, is characterized by the propagation matrix [42]

$M_{\gamma}=\left[\begin{array}{cc}\exp \left(-i k_{z \gamma} d_{\gamma}\right) & 0 \\ 0 & \exp \left(i k_{z \gamma} d_{\gamma}\right)\end{array}\right]$, where $d_{\gamma}$ is the thickness of the respective material. These matrices can be found by applying the Maxwell's boundary conditions, for each layer, considering a incident plane wave from a transparent medium $C$ (see Fig. 1). Below we will perform this calculation.

For TE waves, we assume that, for a given generation of the Octonacci sequence, the electrical and magnetic fields for $\mathrm{N}$-th 
layers (where $N=0,1,2, \ldots, n$, and $n$ is the total number of slabs in the unit cell) have the form,

$\vec{E}_{j}^{(N)}=\left(0, E_{y j}^{(N)}, 0\right)$,

$E_{y j}^{(N)}=\left[A_{1 j}^{(N)} \exp \left(i k_{z j} z\right)+A_{2 j}^{(N)} \exp \left(-i k_{z j} z\right)\right] \exp \left(i k_{x} x-i \omega t\right)$,

where $A_{1 j}^{(N)}$ and $A_{2 j}^{(N)}(j=A$ or $B ; N=0,1,2 \ldots)$, are their amplitudes. Here we have considered, without loss of generality, the magnetic permeability of each material equal to one, i.e., $\mu_{j}=1$. On the other hand, for the case of TM waves, we assume that the magnetic field for $\mathrm{N}$-th slab is given by

$\vec{H}_{j}^{(N)}=\left(0, H_{y j}^{(N)}, 0\right)$

$H_{y j}^{(N)}=n_{j}\left[A_{1 j}^{(N)} \exp \left(i k_{z j} z\right)-A_{2 j}^{(N)} \exp \left(-i k_{z j} z\right)\right] \exp \left(i k_{x} x-i \omega t\right)$

where $n_{j}$ is the refraction index in the medium $j$. Since we have considered $\mu_{j}=1$, the impedance term is $\sqrt{\mu_{j} / \epsilon_{j}}=\sqrt{1 / \epsilon_{j}}=1 / n_{j}$ and the magnetic field $\vec{B}=\vec{H}$. This impedance term appears on denominator of Eq. (11), due to definition of $\vec{H}=-(\vec{\nabla} \times \vec{E}) /(i \omega \mu)$.

Application of Maxwell's electromagnetic boundary conditions (continuity of the tangential components of $\vec{E}$ and $\vec{D}$ for TE waves, or, tangential components of $\vec{E}$ and $\vec{H}$ for TM waves) at the interfaces $C|B, B| A, A \mid B \ldots$, and finally $B \mid C$ (see Fig. 1 ) yields:

(a) at the interface $C \mid B$ (considering $z=0$ at the interface between the layer $B$ and the surrounding medium $C$ ), we have
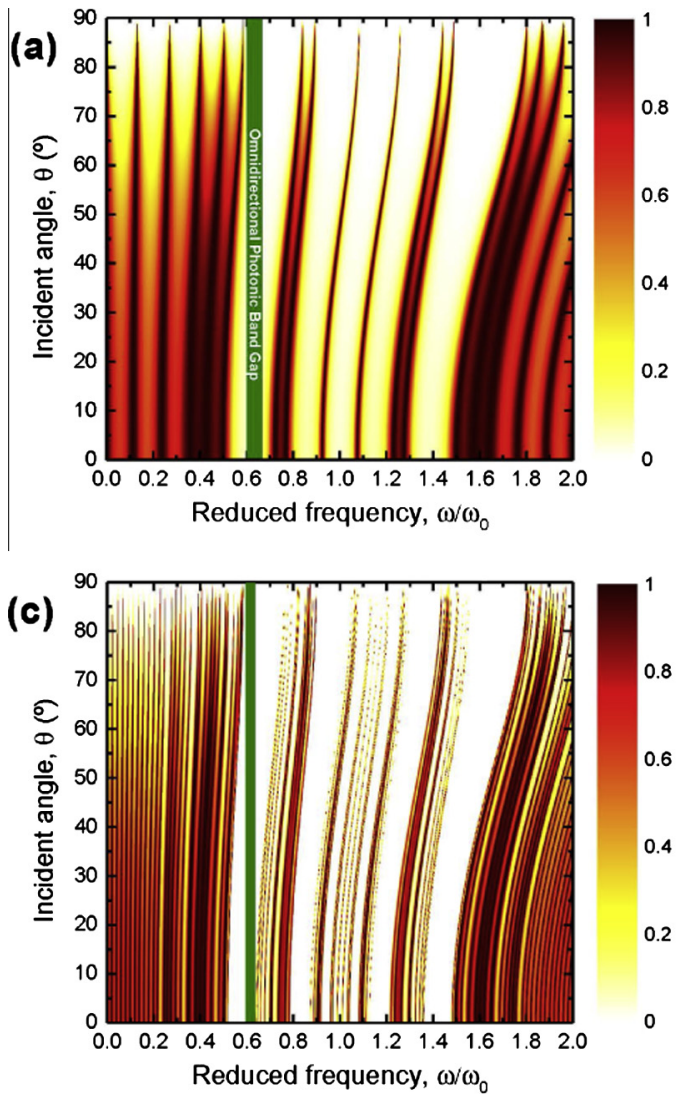

$$
\left(\begin{array}{l}
A_{1 C}^{(0)} \\
A_{2 C}^{(0)}
\end{array}\right)=M_{C B}\left(\begin{array}{l}
A_{1 B}^{(1)} \\
A_{2 B}^{(1)}
\end{array}\right)
$$

(b) at the interface $B \mid A$ (considering $z=d_{B}$ at the interface between the layers $B$ and $A$ ), we find

$$
\left(\begin{array}{c}
A_{1 B}^{(1)} \\
A_{2 B}^{(1)}
\end{array}\right)=M_{B} M_{B A}\left(\begin{array}{c}
A_{1 A}^{(3)} \\
A_{2 A}^{(3)}
\end{array}\right)
$$

The successive applications of the transmission and propagation matrices along the finite structure gives

$$
\left(\begin{array}{l}
A_{1 C}^{(0)} \\
A_{2 C}^{(0)}
\end{array}\right)=M_{C B A B B B C}\left(\begin{array}{c}
A_{1 C}^{(N)} \\
0
\end{array}\right)=M_{n}\left(\begin{array}{c}
A_{1 C}^{(N)} \\
0
\end{array}\right)
$$

where the transfer-matrix for the $n$-th generation $M_{n}$ is a $2 \times 2$ matrix given by

$M_{n}=M_{C B A B \ldots B C}=M_{C B} M_{B} M_{B A} M_{A} \cdots M_{B} M_{B C}$

Now, by considering the iterative rule given in Eq. (1), we are capable to find the following general formula for the transfer-matrices in the Octonacci quasiperiodic system,

$M_{n}=M_{C B} T_{n} M_{B C}, \quad($ for $n \geqslant 3)$

where

$T_{n}=T_{n-1} T_{n-2} T_{n-1}$,

whose initial conditions are $M_{1}=M_{C B} T_{1} M_{B C}$, $M_{2}=M_{C B} T_{2} M_{B C}, T_{1}=M_{B}$, and $T_{2}=M_{B} M_{B A} M_{A} M_{A B} M_{B}$.
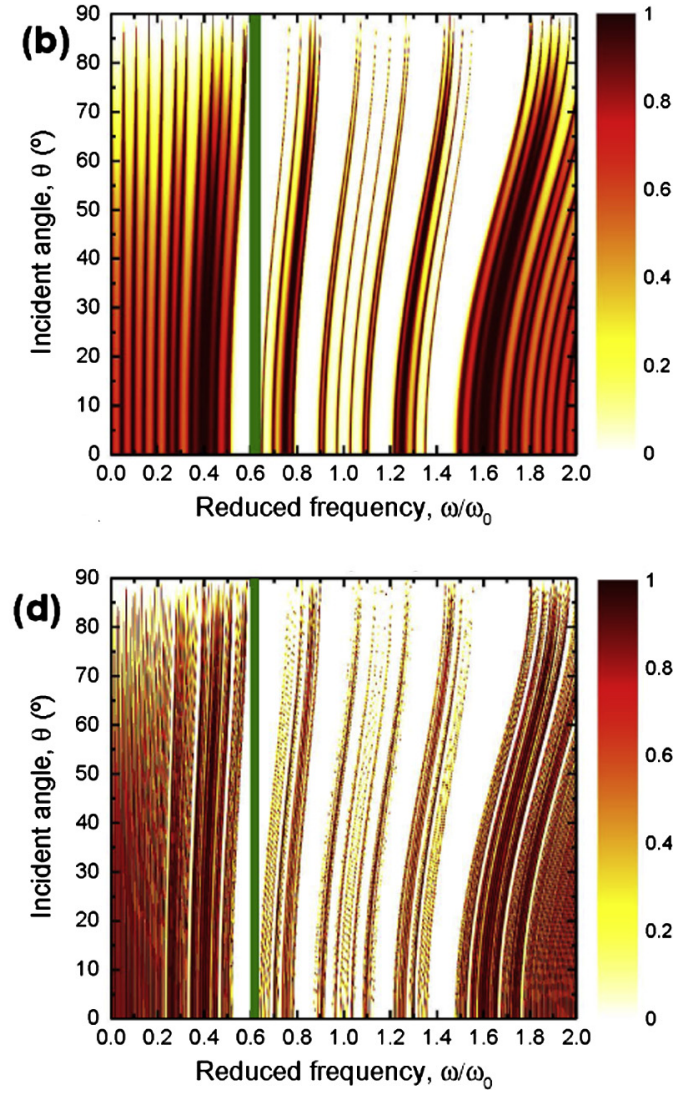

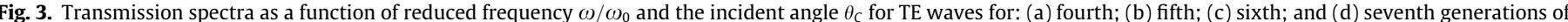

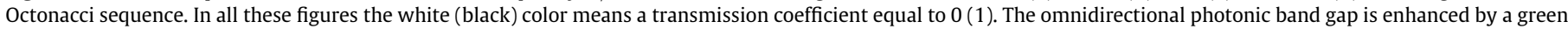
rectangle. (For interpretation of the references to color in this figure legend, the reader is referred to the web version of this article.) 


\section{Numerical results}

Now we present our numerical results to illustrate the optical transmission spectra of some quasiperiodic structures. We consider the same physical parameters used in Ref. [35], i.e., those appropriated for silicon dioxide $(A)$ and titanium dioxide $(B)$; they are virtually absorption-free above $400 \mathrm{~nm}$ [43]. Also, we consider the individual layers as quarter-wave layers, for which the quasiperiodicity is expected to be more effective [36], with the central wavelength $\lambda_{0}=700 \mathrm{~nm}$. These conditions yield the physical thickness $d_{\gamma}=\left(700 / 4 n_{\gamma}\right) \mathrm{nm}, \gamma=A$ or $B$, such that $n_{A} d_{A}=n_{B} d_{B}$. Their dielectric constants around the central wavelength $\lambda_{0}=700 \mathrm{~nm}$ are $n_{A}=1.45$ and $n_{B}=2.30$, respectively. We also consider medium $C$ to be vacuum, and the phase shifts are given by: $\delta_{A}=(\pi / 2) \Omega \cos \left(\theta_{A}\right), \delta_{B}=(\pi / 2) \Omega \cos \left(\theta_{B}\right)$, where $\Omega$ is the reduced frequency $\omega / \omega_{0}=\lambda_{0} / \lambda$ [35].

The light transmission spectrum, as a function of reduced frequency $\omega / \omega_{0}$, for the fifth generation of the quasiperiodic Octonacci sequence (where $P_{5}=17$ layers) is presented in Fig. 2a, for TE waves and a normally incident wave, i.e., $\theta_{C}=90^{\circ}$. As it is expected for normal incidence, the transmission spectrum has a mirror symmetry around the mid-gap reduced frequency $\omega / \omega_{0}=1$ because the quarter-wavelength condition for a periodic multilayer was taken in account. However, differently of what Vasconcelos and Albuquerque reported about a Fibonacci photonic quasicrystal [35], the light is almost entirely reflected and the structure is quite opaque (the transmission coefficient is less than 0.02 for both wave polarizations) at that frequency. This happens because, from a wave point of view, the layers $A$ and $B$ are not equivalent. The transmission spectrum for TM waves is not displayed here because it is qualitatively and quantitatively equal to the TE case.

Furthermore, as usually occur for any wave phenomena in quasiperiodic heterostructures, the transmission spectrum presents a scaling property with respect to the generation number of Octonacci sequence, within a symmetrical interval around the mid-gap reduced frequency $\omega / \omega_{0}=1$. To make this more clear, we present in Fig. 2b the transmission spectra shown in Fig. 2a, but for the reduced range of frequency. This spectrum is similar to the one representing the sixth generation of the quasiperiodic Octonacci sequence (where $P_{6}=41$ layers), displayed in Fig. 2c for the range of frequency reduced by a scale factor approximately equal to 2.6. In Fig. $2 \mathrm{~d}$ and e, we present the transmission spectra for seventh and eighth generations (where $P_{7}=99$ and $P_{8}=239$ layers), and we can observe that such spectra repeat every generation for a Octonacci unidimensional photonic quasicrystal, while in the Fibonacci sequence case, the spectra repeat each six generations, when the optical parameters of medium $C$ are different from $A$ [35], and each three generations, when we take medium $C$ equal to $A$ one [26]. This scaling property corresponds to a self-similar behavior of the spectrum, which is a qualitative evidence of a fractal spectrum.

In Fig. 3, we have the transmission coefficient versus the reduced frequency $\omega / \omega_{0}$ and the incident angle $\theta_{C}$ for 4 th, 5 th, 6th and 7th generations (Fig. 3a, b, c and d), respectively, for TE polarization. On the other hand, in Fig. 4 we have the same as Fig. 3, but for TM polarization. Only for TE waves we could observe a narrow region with a well defined photonic band gap, enhanced by the green rectangle. Beside of this, the band gaps are independent of the incident angle, and are all localized at, approximately,
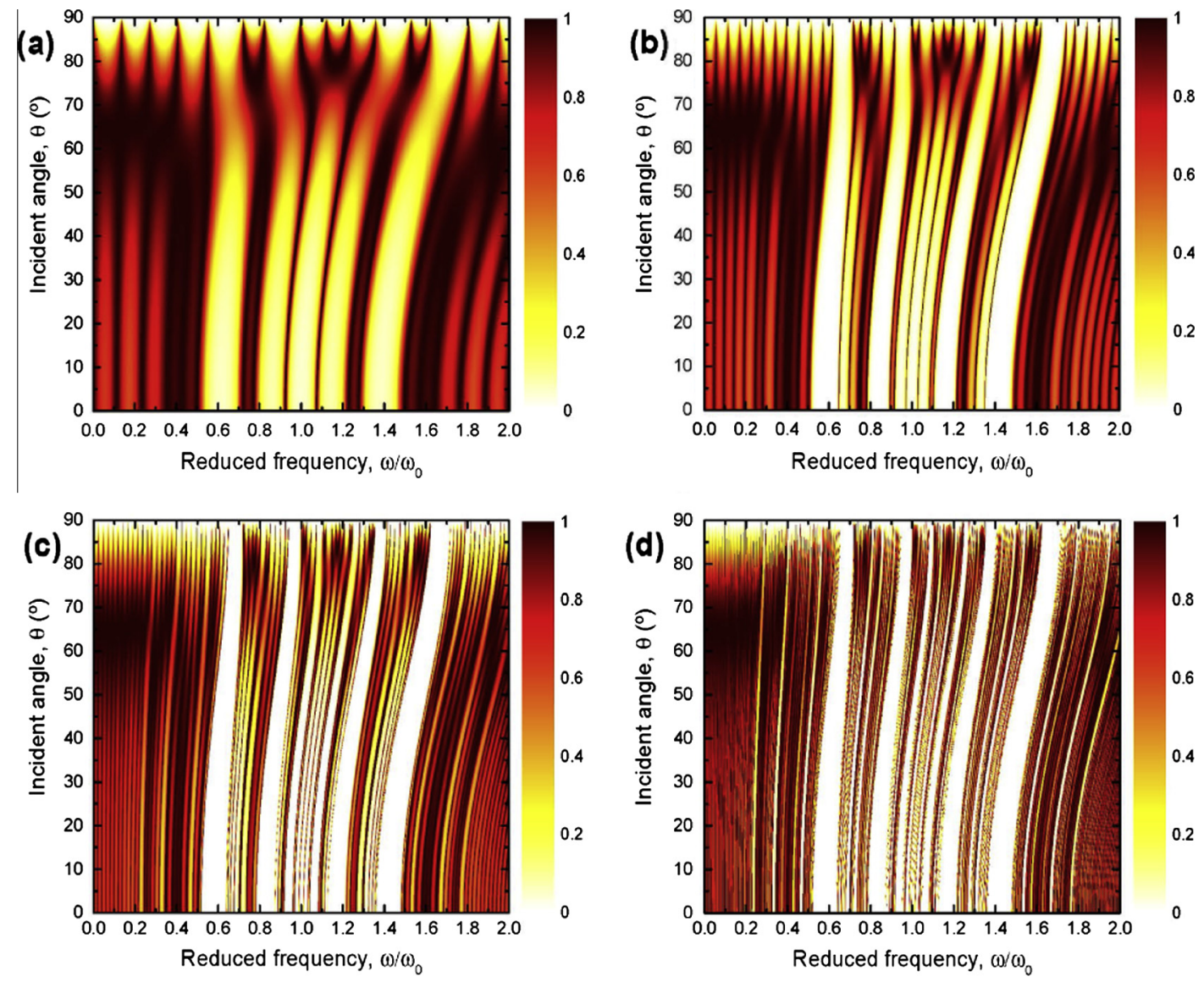

Fig. 4. Same as Fig. 3, but now for TM waves (p-polarization). For this case, a well defined omnidirectional photonic band gap does not appear. 
the same reduced frequency region $\left(0.6 \leqslant \omega / \omega_{0} \leqslant 0.65\right)$ and they have almost the same width. Clearly, the appearing of these band gaps are due to the long-range order of the arrangements of the layers in the Octonacci lattice, and this long-range order is responsible for the anomalous interference, giving rise to these omnidirectional band gaps.

\section{Conclusions}

In summary, in this work we calculated the transmission spectra for photonic crystals generated in accordance to Octonacci sequence. Firstly, for normally incident wave, we observe that, for a same generation, the transmission spectra for both TE and TM waves are equal, at least qualitatively, and they present a scaling property where a self-similar behavior is obtained as a qualitative evidence of these spectra are fractals. Our results also show the spectra regions where the omnidirectional band gaps emerges for specific generations of Octonacci photonic structures, except to TM waves. For TE waves, we note that all of them have almost the same width, for different generations. Also, the localization of modes is enhanced, as we increase the generation number, as it was expected [13]. This property could serve as a tool for sensing devices, since the localization can be controlled through physical parameters as the thickness of the layers [44]. In this case, it is expected that the quasiperiodicity could affect in someway the transmission spectra, creating well defined band gaps and a self-similar pattern as in Fibonacci multilayered photonic structure [35].

There are many applications of such type of systems in optical system and communications. For example, recently several authors have studied one dimensional quasicrystals in order to understand the passband [45], absorption [46], and omnidirectional reflection [40] properties in Fibonacci (generalized in last case) quasicrystals. Besides the self-similarity, they have found that is possible to control the bandgap properties by constructing the photonic crystal heterostructure with photonic crystals and quasi-crystals [45]. By using the effective medium theory, Xu et al. [45] have concluded that is possible to understand the passband of the photonic crystal and quasi-crystal, and the optimal lengths for the maximum bandgap and phase matching condition can be obtained. On the other hand, the study of the absorption properties in one dimensional quasiperiodic photonic crystals composed by a thin film metallic layer and dielectric Fibonacci multilayer, have resulted to almost perfect absorption for the energy of the incident wave, by tuning the length of the dielectric layer together with the high refractive index [46], making it possible to design a perfect absorber with channel number as high as twelve and bandwidth as narrow as $1 \mathrm{~nm}$, which is very attractive in sensor/detector technology and highly integrated dense wavelength division multiplexing networks. Also, the omnidirectional reflection is possible in generalized Fibonacci quasicrystals by controlling the thickness [40]. Regarding these generalized sequences, other ideas could be extended to photonic crystals generated in accordance to the Octonacci sequence studied here, namely, diffusive and spectral properties [39], clustering of energy spectra [31] and quantum diffusion [37]. We hope that our theoretical predictions can be tested through experimental measurements in further applications and researches.

\section{Acknowledgements}

The authors would like to thank CAPES and CNPq (Brazilian Science Funding Agencies) for the financial support.

\section{References}

[1] J.E. Yablonovitch, Phys. Rev. Lett. 58 (1987) 2059-2062.

[2] S. John, Phys. Rev. Lett. 58 (1987) 2486-2489.

[3] D.W. Prather, A. Sharkawy, S. Shi, J. Murakowski, G. Schneider, Photonic Crystals: Theory, Applications and Fabrication, Wiley, United States, 2009.

[4] V.E. Laine (Ed.), Photonic Crystals: Fabrication, Band Structure and Applications, Nova Science Pub. Inc., United States, 2010.

[5] A. Massaro (Ed.), Photonic Crystals - Introduction, Applications and Theory, InTech, Croatia, 2012.

[6] K. Sakoda, Optical Properties of Photonic Crystals, 2nd ed., Springer, Berlin Heidelberg, Berlin, 2004.

[7] J.D. Joannopoulos, S.G. Johnson, J.N. Winn, R.D. Meade, Photonic Crystals: Molding the Flow of Light, 2nd ed., Princeton University Press, New Jersey, 2008.

[8] A. Cerqueira Jr., Rep. Prog. Phys. 73 (2010) 024401.

[9] E. Maciá, Rep. Prog. Phys. 75 (2012) 036502.

[10] P. Kramper, M. Agio, C.M. Soukoulis, A. Birner, F. Muller, R.B. Wehrspohn, U. Gosele, V. Sandoghdar, Phys. Rev. Lett. 92 (2004) 113903.

[11] D.N. Chigrin, A.V. Lavrinenko, D.A. Yarotsky, S.V. Gaponenko, Appl. Phys. A: Mater. Sci. Process. 68 (1999) 25-28.

[12] D. Shechtman, I. Blech, D. Gratias, J.W. Cahn, Phys. Rev. Lett. 53 (1984) 1951.

[13] E.L. Albuquerque, M.G. Cottam, Polaritons in Periodic and Quasiperiodic Structures, Elsevier, Amsterdam, 2004.

[14] Page of 2011 Nobel Prize in Chemistry awarded to Dan Shechtman. <http:// www.nobelprize.org/nobel_prizes/chemistry/laureates/2011/press.html>.

[15] H. Bohr, Jahresbericht der Deutschen Mathematiker-Vereinigung 34 (1926) $25-40$.

[16] W. Steurer, S. Deloud, Crystallography of Quasicrystals, Concepts, Methods and Structures, Springer, Berlin, Heidelberg, Berlin, 2009.

[17] R. Penrose, Bull. Inst. Math. Appl. 10 (1974) 266-271.

[18] B. Grünbaum, G.C. Shephard, Tilings and Patterns, W.H. Freeman, New York, 1986.

[19] R. Ammann, B. Grünbaum, G.C. Shephard, Discrete Comput. Geom. 8 (1992) 125.

[20] P. Kramer, R. Neri, Acta Crystallogr. A 40 (1984) 580-587.

[21] R. Towle, Colored Zonotiles, from Wolfram Library Archive. <http:// library.wolfram.com/infocenter/MathSource/1197/>.

[22] E. Maciá, Rep. Prog. Phys. 69 (2006) 397-441.

[23] W. Steurer, D.S. Widmer, J. Phys. D: Appl. Phys. 40 (2007) R229-R247.

[24] Z.V. Vardeny, A. Nahata, A. Agrawal, Nat. Photonics 7 (2013) 177-187.

[25] C.H.O. Costa, P.H.R. Barbosa, F.F. Barbosa Filho, M.S. Vasconcelos, E.L. Albuquerque, Solid State Commun. 150 (2010) 2325-2328.

[26] M. Kohmoto, B. Sutherland, K. Iguchi, Phys. Rev. Lett. 58 (1987) 2436-2438.

[27] T. Zentgraf, A. Christ, J. Kuhl, N.A. Gippius, S.G. Tikhodeev, D. Nau, H. Giessen, Phys. Rev. B 73 (2006) 115103.

[28] T. Utikal, T. Zentgraf, S.G. Tikhodeev, M. Lippitz, H. Giessen, Phys. Rev. B 84 (2011) 075101.

[29] E.M. Barber (Ed.), Aperiodic Structures in Condensed Matter: Fundamentals and Applications, CRC Press, 2008.

[30] L. dal Negro (Ed.), Optics of Aperiodic Structures: Fundamentals and Device Applications, Pan Stanford Publishing, 2013.

[31] G.Y. Oh, C.S. Ryu, M.H. Lee, Phys. Rev. B 47 (1993) 6122.

[32] C.A.A. Araújo, M.S. Vasconcelos, P.W. Mauriz, E.L. Albuquerque, Opt. Mater. 35 (2012) 18-24.

[33] C.H.O. Costa, M.S. Vasconcelos, J. Phys.: Condens. Ma. 25 (2013) 286002.

[34] C.H. Costa, M.S. Vasconcelos, J. Appl. Phys. 115 (2014) 17C115.

[35] M.S. Vasconcelos, E.L. Albuquerque, A.M. Mariz, J. Phys.: Condens. Mat. 10 (1998) 5839-5849.

[36] M.S. Vasconcelos, E.L. Albuquerque, Phys. Rev. B 59 (1999) 11128-11131.

[37] H.Q. Yuan, U. Grimm, P. Repetowicz, M. Schreiber, Phys. Rev. B 62 (2000) $15569-15578$

[38] J.P. Lu, T. Odagaki, J.L. Birman, Phys. Rev. B 33 (1986) 4809-4817.

[39] V.Z. Cerovski, M. Schreiber, Phys. Rev. B 72 (2005) 054203.

[40] A.G. Barriuso, J.J. Munzón, T. Yonte, A. Felipe, L.L. Sánchez-Soto, Opt. Exp. 21 (2013) 30039.

[41] S. Golmohammadi, M.K. Moravvej-Farshi, A. Rostami, A. Zarifkar, Prog. Electromagn. Res. 75 (2007) 69-84.

[42] T. Zhan, X. Shi, Y. Dai, X. Liu, J. Zi, J. Phys.: Condens. Mat. 25 (2013) 215301.

[43] W. Gellermann, M. Kohmoto, B. Sutherland, P.C. Taylor, Phys. Rev. Lett. 72 (1994) 633-636.

[44] S. Barth, F.H. Ramirez, J.D. Holmes, A.R. Rodriguez, Prog. Mater. Sci. 55 (2010) 563-627.

[45] S. Xu, Y. Zhu, L. Wang, P. Yang, P.K. Chu, J. Opt. Soc. Am. B 31 (2014) 664-671.

[46] Y. Gong, X. Liu, L. Wang, H. Lu, G. Wang, Opt. Exp. 19 (2011) 9759-9769. 